\title{
Inaccurate Blood Pressure Readings in the Intensive Care Unit: An Observational Study
}

Brian Tse ${ }^{1}$, Mark A. Burbridge ${ }^{1}$, Richard A. Jaffe ${ }^{1}$, John Brock-Utne ${ }^{1}$

1. Anesthesiology, Stanford University Medical Center, Stanford, USA

Corresponding author: Mark A. Burbridge, markburb@stanford.edu

\begin{abstract}
Measuring and monitoring cerebral perfusion pressure (CPP) is important in the management of patients with certain neurological conditions. To accurately reflect blood pressure at the circle of Willis, the arterial line transducer should be leveled at the tragus. This study measured the relative distance of the transducer to the tragus in 100 intensive care unit (ICU) patients in the mixed ICU at our institution, of which 44 patients had a pressure-sensitive neurological diagnosis. For neurological patients, the average distance was $10.9 \mathrm{~cm}$ and for non-neurological patients, the average distance was $11.4 \mathrm{~cm}$ (p-value: 0.60$)$. This suggests that the arterial line transducer was leveled at approximately the same level regardless of pathology, potentially leading to falsely elevated CPP readings in patients with pressure-sensitive neurological pathology.
\end{abstract}

Categories: Anesthesiology, Neurology, Neurosurgery

Keywords: cerebral perfusion, intensive care, arterial line

\section{Introduction}

The arterial line is a critically important monitoring tool used in the operating room and intensive care unit (ICU) in order to obtain a continuous measurement of arterial blood pressure (ABP) and its waveform. Regardless of the location of the intra-arterial cannula, the vertical height of the pressure transducer measures the ABP at that vertical point of the body [1]. In most general ICUs, ABP transducers are leveled at the phlebostatic axis, the reference point for the right atrium, using the mid-axillary line at the level of the fourth intercostal space as external landmarks [2].

In patients with certain neurological conditions, the cerebral perfusion pressure (CPP) is an important parameter that directly correlates with cerebral blood flow, and therefore brain perfusion. Its measurement in clinical practice is calculated by the difference between mean arterial pressure (MAP) and intracranial pressure (ICP), or central venous pressure (CVP), if higher [3]. In order to manage CPP for various clinical scenarios, the accurate measurement of MAP is essential. Although many international guidelines have published and studied target values for CPP, none have specified how to measure the MAP for the CPP calculation until 2015, when the Neuroanaesthesia Society of Great Britain and Ireland (NASGBI) and Society of British Neurological Surgeons (SBNS) issued a joint position statement. They recommend that the arterial transducer be leveled at the tragus of the ear or external auditory meatus, which corresponds to the level of the middle cranial fossa and circle of Willis [4]. They also recommend the arterial transducer be repositioned, to remain level with the tragus, after changes in head elevation.

๑) Copyright 2018

Tse et al. This is an open access article distributed under the terms of the Creative Commons Attribution License CC-BY 3.0., which permits unrestricted use, distribution, and reproduction in any medium, provided the original author and source are credited.
In the mixed ICU of our quaternary care academic medical center, nurses are trained in maintenance of arterial lines and are responsible for the transducer height. It has been observed that the level of the transducer is transduced at the phlebostatic axis, even when CPP is of importance because no protocol exists to make height adjustments in patients who have pressure-sensitive neurological conditions. The goal of this study is to measure the relative height of arterial line transducers compared to the tragus.

\section{Materials And Methods}

This study started as a quality improvement project and was reviewed by the local Institutional Review Board (IRB) and the requirement for written informed consent was waived by the IRB. Patients included in this observational study were admitted to the Stanford University Hospital Medical and Surgical ICU between September 2017 and October 2017. This is a mixed ICU that has no physical division based on patient pathology, and does not have a dedicated neurosciences ICU. The inclusion criterion is the active measurement of ABP from an arterial line. The eligible patients were identified via electronic medical record (Epic Systems, Madison, WI). Each patient was evaluated at the bedside, where the height of the arterial line transducer relative to the tragus was measured as well as the degree of elevation of the head of bed position.

The ABP transducer height was measured using a string line level that originated from the patient's tragus and extended to the pole of the ABP transducer. The height difference from the level of the tragus on the pole to the transducer was measured with a ruler and recorded. The head of bed position, indicated in 


\section{Cureus}

degrees on the hospital bed, was recorded as well.

Each patient's primary ICU diagnosis and active secondary diagnoses were collected and categorized as a neurological diagnosis or not. The neurological diagnoses included any neurological surgery (tumor resection, aneurysm clipping, vascular malformation resection), ischemic stroke, intracranial hemorrhage, hypoxic brain injury, traumatic brain injury (TBI), and/or encephalitis/meningitis. Basic descriptive statistics and a student's $t$-test between the transducer height of the two groups were appropriately calculated.

\section{Results}

Data from 100 patients with arterial lines were collected in the month-long observational period. There were 44 patients with neurological diagnoses and 56 in the non-neurological group. The average height of the transducer for all patients was $11.2 \mathrm{~cm}$ below the tragus. For neurological patients, the average height was $10.9 \mathrm{~cm}$ below the tragus and for non-neurological patients, the average height was $11.4 \mathrm{~cm}$ below the tragus. The difference in height between the two groups was $0.44 \mathrm{~cm}$ with a p-value of 0.60 (Figure 1).

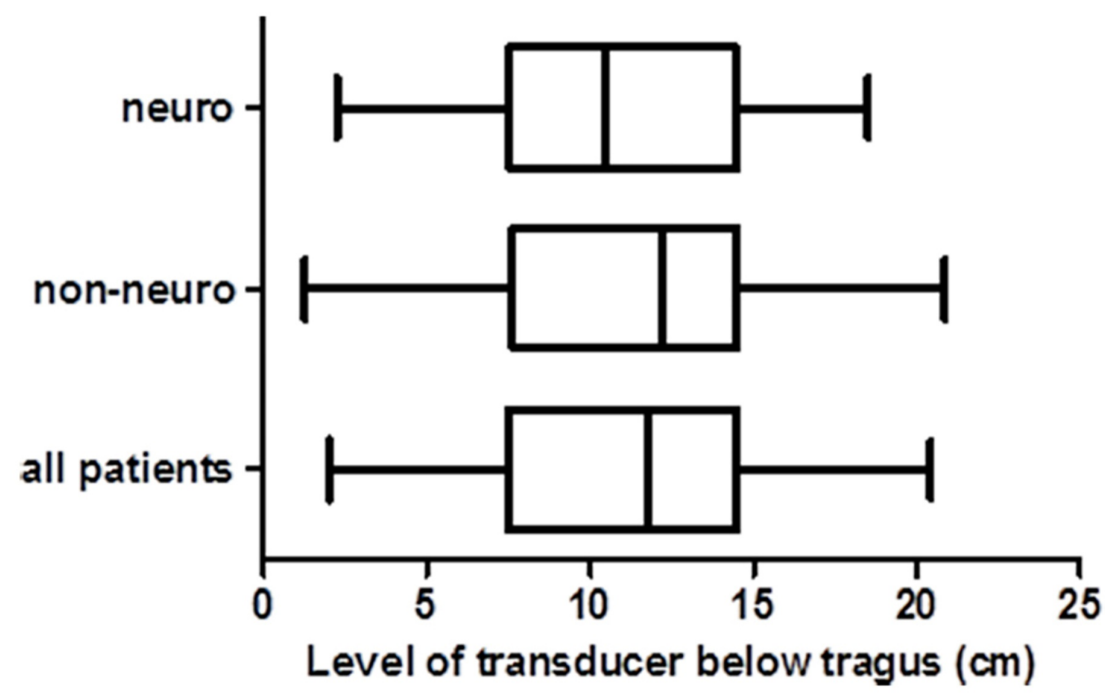

FIGURE 1: Box plots of transducer distances.

Box plots of the distance of the transducer below tragus for patients with neurological diagnoses, nonneurological diagnoses, and for all patients.

A scatter plot of the distance of the transducer below the tragus to the angle of the bed shows a positive correlation, with a Pearson's $r$ value of 0.65 . This indicates that in general, a higher angle of the head of bed correlates with a greater distance of the arterial line transducer below the tragus (Figure 2). 


\section{Cureus}

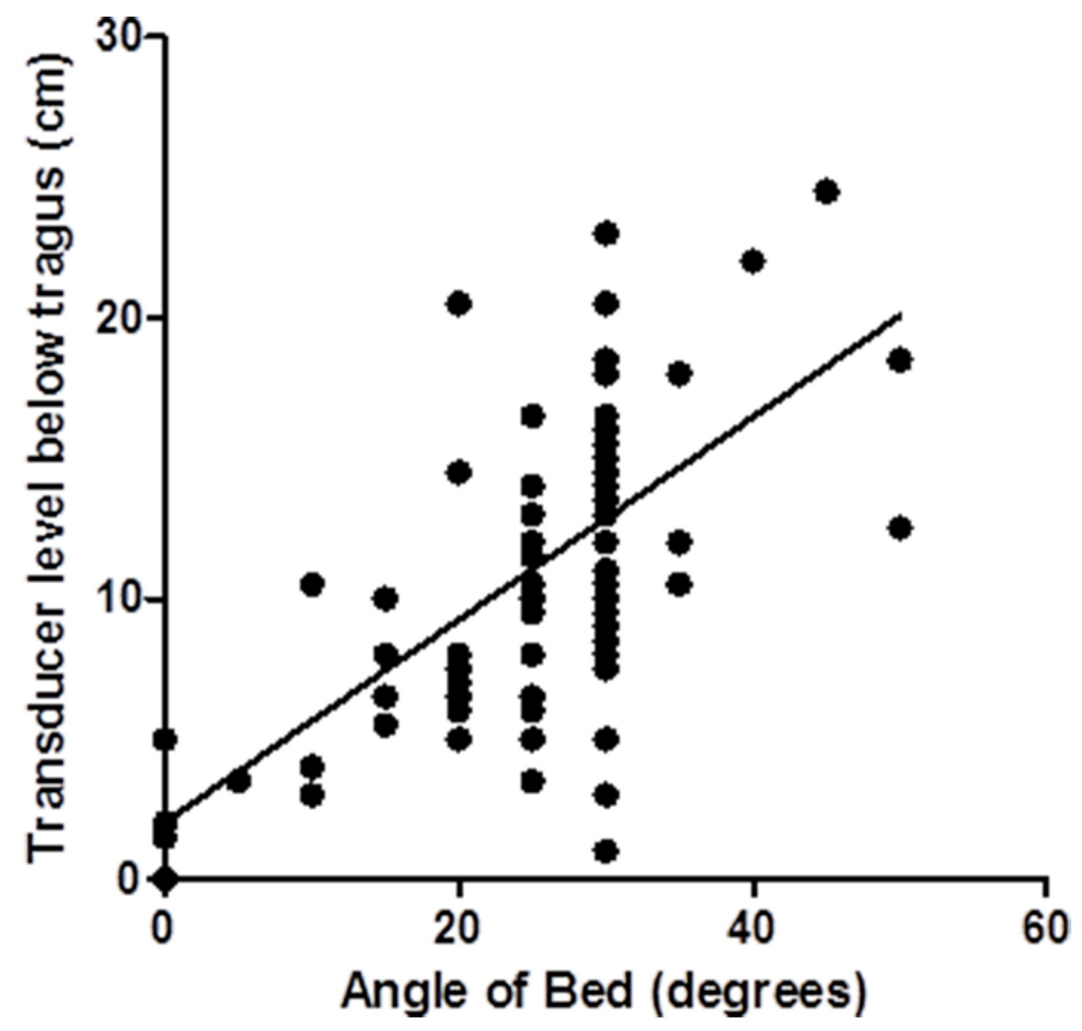

FIGURE 2: Transducer height relative to bed angle.

Scatter plot of the transducer level and the angle of the bed with least squares regression line for all patients.

\section{Discussion}

This observational study showed that arterial line transducers in our ICU are leveled at approximately the same height below the tragus in patients with and without pressure-sensitive neurological pathologies. It also showed a negative correlation between the angle of the head of the bed and the distance of the transducer below the tragus. This suggests that at our institution, the transducer is leveled at the phlebostatic axis for all ICU patients, regardless of their diagnosis. It also demonstrates that as the angle of the head of the bed is increased, the discrepancy between MAP and CPP increases presumably because the arterial line transducer is not re-adjusted, and remains at the phlebostatic axis.

The clinical implication of this discrepancy is an inaccurate MAP, and therefore CPP reading, when the patient's head is elevated. For instance, for a vertical distance of $10 \mathrm{~cm}$, the corresponding change in MAP is approximately $7.5 \mathrm{mmHg}$. Using this study's finding of an average distance of the transducer being $11 \mathrm{~cm}$ below the tragus, that equates to a MAP that should be $8 \mathrm{mmHg}$ lower, which decreases the CPP measurement by $8 \mathrm{mmHg}$. For critically ill patients, this could potentially result in cerebral hypoperfusion and ischemia. It could also represent a critical issue in patients with vasocclusive disease such as Moyamoya disease whose cerebral perfusion is dependent on accurate MAP parameters at the risk of inducing transient ischemic attacks, strokes, or death. Moreover, patients who experience intra-operative neuromonitoring changes reflecting impending neurological compromise must often be maintained in a strict range of accurate MAPs [5-6].

We acknowledge this study is an observational study with no clinical outcomes measured. The validity of CPP directed management for TBI is also debatable, given the lack of level 1 evidence supporting it [7]. However, this could in part be due to the lack of consistency amongst studies attributed to the lack of standardization of transducer prior to the NASGBI and SBNS guidelines. Moreover, we also acknowledge that our patients may also have other clinical indications for ABP monitoring at the phlebostatic axis that may necessitate an additional transducer or arterial cannulation.

\section{Conclusions}

In conclusion, our study shows that without placing the arterial line transducer at the tragus to better approximate CPP, major discrepancies can result, which is exacerbated by increasing the angle of the 
patient's bed. We are currently engaged in discussions with our ICU leadership to educate and protocolize moving the height the ICU transducer to the tragus for indicated neurological pathologies, and would recommend other such ICUs to do the same.

\section{Additional Information \\ Disclosures}

Human subjects: Consent was obtained by all participants in this study. Animal subjects: All authors have confirmed that this study did not involve animal subjects or tissue. Conflicts of interest: In compliance with the ICMJE uniform disclosure form, all authors declare the following: Payment/services info: All authors have declared that no financial support was received from any organization for the submitted work. Financial relationships: All authors have declared that they have no financial relationships at present or within the previous three years with any organizations that might have an interest in the submitted work. Other relationships: All authors have declared that there are no other relationships or activities that could appear to have influenced the submitted work.

\section{References}

1. McCann UG, Schiller HJ, Carney DE: Invasive arterial BP monitoring in trauma and critical care: effect of variable transducer level, catheter access, and patient position. Chest. 2001, 120:1322-1326.

2. Winsor T, Burch GE: Phlebostatic axis and phlebostatic level: reference levels for venous pressure measurement in man. Proc Soc Exp Biol. 1945, 58:165-169.

3. Smith M: Cerebral perfusion pressure. Br J Anaesth. 2015, 115:488-490. 10.1093/bja/aev230

4. Thomas, E, on behalf of NACCS, Czosnyka, M, Hutchinson P, and on behalf of SBNS: Calculation of cerebral perfusion pressure in the management of traumatic brain injury: joint position statement by the councils of the Neuroanaesthesia and Critical Care Society of Great Britain and Ireland (NACCS) and the Society of British Neurological Surgeons (SBNS). Br J Anaesth. 2015, 115:487-488. 10.1093/bja/aev233

5. Brain Trauma Foundation, American Association of Neurological Surgeons, Congress of Neurological Surgeons: . Guidelines for the management of severe traumatic brain injury . J Neurotrauma. 2007, 24:1-106.

6. Kosty JA, LeRoux PD, Levine J: Brief report: a comparison of clinical and research practices in measuring cerebral perfusion pressure: a literature review and practitioner survey. Anesth Analg. 2013, 117:694-698. 10.1213/ANE.0b013e31829cc765

7. Kirkman MA, Smith M: Intracranial pressure monitoring, cerebral perfusion pressure estimation, and ICP/CPP-guided therapy: a standard of care or optional extra after brain injury?. Br J Anaesth. 2014, 112:3546. $10.1093 / \mathrm{bja} /$ aet418 